\title{
A Prototype Of Virtually Interactive Hand Activating Devise - Low Cost Portable Head Mounted System (VIHAD Plus) For Neurological Rehabilitation
}

\author{
10.5339/qfarc. 2014.HBPP0446
}

Ajimsha Mohammed Sharafudeen, Phd; John John Cabibihan; Uvais Ahmed Qidwai; Lina Majed

\section{Corresponding AUTHOR :}

ajimshaw.ms@gmail.com

Hamad Medical Corporation, Doha, Qatar

\section{$\underline{\text { Abstract }}$}

\section{ABSTRACT}

Background and Purpose: Restoring function in individuals who have severe paralysis of the upper extremity secondary to stroke is challenging. Recent technologies have made it possible to use robotic devices as novel tools for assisting the therapists to provide safe and intensive rehabilitation with repeated motions. However, most of the training robots are types of Continuous Passive Motion (CPM) devices that produce slower and stereotyped movement patterns. Earlier works have shown that passive or slow movements do not significantly benefit motor improvement. Several studies reveal that even the use of ipsilateral electromyographic (EMG) pattern recognition approaches might not be practical to decode movement intention and, may negatively affect re-mapping of the neural pathways in the brain. To have a successful hand rehabilitation system, the system should be able to produce a wide variety of unpredicted and challenging movement patterns of various degrees of speed and range of motion with increasing complexity, sufficient enough to produce the necessary neurological plasticity of the affected brain. Current rehabilitation devices are not sufficient to produce such a range of complex activities which enables maximum neurological plasticity.

Objective: In this work, we describe a prototype of the contralateral EMG-based Interactive Hand Activating Devise for Stroke (IHADS) system that can detect a hemiplegic person's intention for bilaterally executed hand activities using his/her surface EMG signals from the non-affected side (contralateral). Furthermore, this system can assist in bilateral hand activities through an exoskeleton attached to the hemiplegic upper extremity to initiate progressively challenging and unpredicted type of activities in a virtual reality (VR) world to obtain optimum functional recovery by inducing maximum neurological plasticity.

Design: The IHADS system is made up of an embedded controller and a robotic exoskeleton, contralateral EMG sensors and a VR interface with a semi-immersed VR system, where the patient will be seeing progressively impulsive activities that would force the brain to activate the affected extremity to manipulate through the remaining neural networks and mirror neuronal system which in turn will optimize the neurological recovery. This means that the paralyzed arm will be following the motion of the healthy arm whose motion is picked up by the EMG sensors and are translated as actuation signals for the exoskeleton to execute virtually created challenging activities.

Conclusion: Contralateral EMG-based 'IHADS' system is a unique, cost effective, highly innovative and portable robotic device. If incorporated into the stroke rehabilitation, this system will be capable of autonomous guidance through the use of real-time feedback from the contralateral upper limb, integrated via the VR interface and the hand activating device to make rehabilitation more intense, functional, motivating, and capable of inducing maximum neurological plasticity.

Keywords: contralateral upper limb EMG, exoskeleton; robot; virtual reality, stroke rehabilitation 